\title{
La motivation des étudiants en sciences de la santé : un levier pédagogique à exploiter et un objet de recherche à construire
}

Le travail publié dans ce numéro par Badre et ses collaborateurs, sur la thématique de la motivation des étudiants en médecine dentaire ${ }^{[1]}$, est de nature à interpeller le lecteur à un double niveau.

Sur le plan de la formation, il tend à confirmer l'impact majeur de l'environnement d'apprentissage des étudiants en sciences de la santé sur leur motivation. Certes, les auteurs se sont inscrits dans une démarche transversale et observationnelle de recueil des données, mais d'autres contributions ont précédemment confirmé le caractère démotivant des études de santé, notamment au cours de la première année ${ }^{[2]}$.

Différents facteurs explicatifs sont couramment évoqués. Nous en citerons deux, qui selon le modèle de la dynamique motivationnelle élaboré par Viau, s'inscrivent respectivement dans le groupe des facteurs de (dé)motivation liés à l'institution - et, notamment, aux décisions relatives à l'organisation des curriculums - et dans celui des facteurs liés à la « classe » (et en particulier aux choix des activités pédagogiques et des activités d'évaluation des apprentissages $)^{[3]}$. Le premier renvoie à la difficulté qu'éprouvent les étudiants à mettre en lien le contenu scientifique de leur première année de formation avec le métier auquel ils sont venus se former, conduisant ainsi à une forme de «désenchantement ${ }^{[2]}$. Le second résulte du caractère hautement compétitif des études de santé, notamment médicales, qui favorise la motivation à réussir, plutôt que la motivation à apprendre $^{[4]}$. Certes, ces deux types de motivation, présentent l'un comme l'autre un intérêt, mais celuici est conditionné au fait que la réussite témoigne de la capacité de l'étudiant à mobiliser des stratégies d'apprentissage élaborées sur le plan cognitif conduisant à des apprentissages couramment dénommés « en profondeur »-, alors même que l'on sait que les modalités d'évaluation, notamment en première année de médecine, n'encouragent pas le recours à de telles stratégies.

C'est ainsi que ces changements, qui affectent les étudiants sur le plan motivationnel et qui sont en particulier induits par les choix en matière d'organisation curriculaire, et par les méthodes et outils d'évaluation privilégiés, conduisent également les intéressés à préférer les stratégies superficielles d'apprentissage, consistant à retenir le contenu de leurs cours par cœur ${ }^{[5]}$. Ces différents constats plaident en faveur de l'impérieuse nécessité d'entreprendre des réformes curriculaires post-flexnériennes, comme elles ont déjà été mises en œuvre dans plusieurs pays, essentiellement en Amérique et en Europe du Nord. C'est ainsi que de nombreux travaux ont démontré les bénéfices, sur le plan motivationnel, des cursus qui visent l'intégration des disciplines cliniques et des sciences biomédicales, notamment ceux construits autour des principes de l'apprentissage par problèmes, dans lesquels des liens sont établis précocement entre les contenus scientifiques à apprendre et la pratique professionnelle 
future des étudiants ${ }^{[6-8]}$. Par ailleurs, plusieurs pratiques d'évaluation des apprentissages sont associées à des effets motivationnels positifs ${ }^{[3,9-13]}$. Il est ainsi établi : que l'on aurait avantage à procéder à des activités d'évaluation en contexte authentique (dont les questions à choix multiples, par exemple, s'éloignent considérablement) ; qu'il serait préférable de recourir à des modalités alternatives à la seule note, qui n'est d'ailleurs bien souvent qu'un score très réducteur, pour codifier les appréciations portées sur les performances; ou encore, qu'il serait souhaitable de recueillir des indicateurs de la qualité des apprentissages qui ne documentent pas uniquement la performance - très réductrice -, mais aussi d'autres aspects du processus d'apprentissage.

Sur le plan de la recherche, l'article de Badre et de ses collaborateurs constitue une opportunité de discuter de la démarche de construction des savoirs autour de la motivation en formation. La motivation est un construit humain qui ne se voit qu'à travers un certain nombre d'indicateurs permettant d'appréhender son niveau chez une personne. Comme tout construit, son appropriation en tant qu'objet d'étude scientifique implique une démarche préalable et obligatoire de conceptualisation, visant à adosser son exploration à l'une des dizaines de théories élaborées dans le cadre des recherches menées dans le domaine de la psychologie et de l'éducation ${ }^{[4]}$. De l'approche psychanalytique freudienne aux conceptions sociocognitivistes, en passant par les courants humaniste - porté notamment par Maslow - et béhavioriste marqué en particulier par les travaux de Hull et de Skinner-, la motivation fait l'objet de recherches scientifiques depuis plus d'un siècle ${ }^{[4]}$. Badre et ses collaborateurs ont choisi d'inscrire leur démarche dans le cadre du courant d'expectation et de la valeur, qui a été investi par des chercheurs comme Lewin, Atkinson, Dweck, Leggett et Nuttin, et selon lequel le comportement d'une personne prend sa source dans la valeur qu'elle attribue au but qu'elle vise et dans le degré de confiance ( expectation ») qu'elle s'accorde afin d'y parvenir avec succès ${ }^{[3,4]}$.

Beaucoup de travaux réalisés sur la motivation des étudiants et des professionnels de la santé revendiquent un ancrage sociocognitiviste, qui permet d'appréhender cette dimension de l'activité humaine à travers les interactions entre les comportements d'une personne, ses caractéristiques individuelles et son environnement ${ }^{[3]}$. C'est notamment le cas des recherches basées sur les théories de l'autodétermination et de la dynamique motivationnelle ${ }^{[3,14]}$. Or, comme pour d'autres thématiques liées à l'éducation des professionnels de la santé, nous constatons que bien trop souvent, les chercheurs novices dans ce domaine décident d'appréhender la motivation selon leurs propres représentations, généralement construites de façon empirique, sans prendre le soin de conceptualiser leur approche. Outre le caractère rédhibitoire de la valorisation scientifique de tels travaux, ce choix «a-théorique » de l'étude de la motivation est doublement critiquable. Sur le plan épistémologique, il mène à la production de résultats dont la validité et la crédibilité sont médiocres. Sur le plan méthodologique, il conduit généralement les chercheurs à construire de novo un questionnaire dont il est impossible de déterminer ce qu'il mesure réellement. Comme en témoigne le travail publié par Badre et ses collaborateurs, la motivation peut se mesurer. Il existe en effet des outils dédiés à cette tâche, dont le nombre élevé s'explique par la diversité des courants de la psychologie et de l'éducation qui se sont efforcés de rendre compte de la motivation. Cette mesure n'a toutefois de sens qu'à partir du moment où ce qui est mesuré a fait l'objet d'une conceptualisation permettant précisément de savoir ce que l'on mesure, puisque la motivation est un concept polysémique dont la construction résulte de l'agrégation d'une multitude de variables correspondant à autant de " sous-dimensions », dont chacune peut être explorée.

Les recherches réalisées avec rigueur sur la motivation des étudiants en sciences de la santé ont permis de produire de nombreux résultats susceptibles de guider les choix des acteurs qui s'inscrivent dans des démarches de (ré)ingénierie de formation et de (ré)ingénierie pédagogique. À titre d'exemple, nous pouvons citer la mise en évidence de liens corrélationnels forts entre le niveau de motivation, la qualité des stratégies d'apprentissage mobilisées par les étudiants, l'attitude de ces derniers face aux situations 
d'échec, leur persévérance et leur performance académique ${ }^{[15-19]}$. Il y a, au regard de ces données, des opportunités remarquables à saisir, dès lors que l'on prend en compte le fait que les étudiants intègrent les facultés de médecine avec des profils motivationnels dits « autodéterminés », c'est-à-dire propices à obtenir de tels effets ${ }^{[5]}$. C'est l'une des raisons ayant conduit des chercheurs, dès la fin des années quatre-vingt-dix, à encourager l'appropriation, par les différentes parties prenantes impliquées dans la formation des étudiants en sciences de la santé, des théories relatives à la motivation, dans le but d'améliorer leurs stratégies d'enseignement et d'évaluation $^{[19]}$.

Afin d'atteindre cet objectif, il est nécessaire de poursuivre les efforts de production de connaissances dans ce domaine, dans la mesure où la motivation demeure un objet récent de recherche dans le champ des sciences de la santé. Un regard sur les travaux réalisés au cours des 20 dernières années permet par exemple de constater le nombre insuffisant d'études reposant sur des démarches qualitatives de recueil et d'analyse des données, un manque d'intérêt pour les indicateurs autres que ceux visant à mesurer la performance académique, et des interrogations persistantes quant à l'influence de certains facteurs contextuels (par exemple, ceux liés à la société) sur la motivation des étudiants en sciences de la santé. Afin de produire des connaissances utilisables à des fins de réflexions éducative et pédagogique, la démarche scientifique qui en est à l'origine doit impérativement s'inscrire dans un cadre théorique - à la fois épistémologique et méthodologique - rigoureux. C'est à cette condition que l'on pourra donner du sens aux résultats obtenus et leur conférer un caractère opératoire susceptible de guider les choix institutionnels et individuels relatifs aux stratégies et modalités de formation des étudiants en sciences de la santé.

Thierry PELACCIA

Centre de formation et de recherche en pédagogie des sciences de la santé

Faculté de médecine,

Université de Strasbourg, France Mailto : pelaccia@unistra.fr

\section{Références}

1. Badre B, Serhier Z, Bennani-Othmani M, El Arabi S. Les étudiants de la faculté de médecine dentaire au Maroc sont-ils motivés? Pédagogie Médicale 2016; 17:109-16.

2. Barker J, Olson J. Medical students' learning strategies: evaluation of first year changes. J Miss Acad Sci 1997;42(2):96-100.

3. Viau R. La motivation en contexte scolaire. Bruxelles : De Boeck, 2009.

4. Pelaccia T, Viau R. Motivation in medical education. AMEE Guide. Med Teach 2016, Sous presse.

5. Kusurkar RA, Ten Cate TJ, van Asperen M, Croiset G. Motivation as an independent and a dependent variable in medical education: a review of the literature. Med Teach 2011;33:e242-62.

6. Pelaccia T, Delplancq H, Triby E, Leman C, Bartier J-C, Dupeyron J-P. La motivation en formation : une dimension réhabilitée dans un environnement d'apprentissage en mutation. Pédagogie Médicale 2008; 9:103-21.

7. Aarnio M, Nieminen J, Pyörälä E, Lindblom-Ylänne S. Motivating medical students to learn teamwork skills. Med Teach 2010;32:e199-204.

8. White CB. Smoothing Out Transitions: How pedagogy influences medical students' achievement of selfregulated learning goals. Adv Health Sci Educ Theory Pract 2007;12:279-97.

9. Holland C. Critical review: medical students' motivation after failure. Adv Health Sci Educ Theory Pract 2016;21:695-710.

10. .Ten Cate TJ, Kusurkar RA, Williams GC. How selfdetermination theory can assist our understanding of the teaching and learning processes in medical education. AMEE guide No. 59. Med Teach 2011;33: 961-73.

11. Mann KV. Motivation in medical education: how theory can inform our practice. Acad Med 1999;74: 237-9.

12. Kusurkar RA, Croiset G, Ten Cate TJ. Twelve tips to stimulate intrinsic motivation in students through autonomy-supportive classroom teaching derived from self-determination theory. Med Teach 2011;33: 978-82. 
13. Stegers-Jager KM, Cohen-Schotanus J, Themmen APN. Motivation, learning strategies, participation and medical school performance. Med Educ 2012; 46:678-88.

14. Deci E, Ryan R. Intrinsic motivation and self-determination in human behavior. New York: Plenum Press, 1985.

15. Fortier MS, Vallerand R, Guay F. Academic motivation and school performance: toward a structural model. Contemp Educ Psychol 1995;20:257-74.

16. Hustinx PWJ, Kuyper H, Werf MPC van der, Dijkstra P. Achievement motivation revisited: new longitudinal data to demonstrate its predictive power. Educ Psychol 2009;29:561-82.

17. Ryan RM, Deci EL. Self-determination theory and the facilitation of intrinsic motivation, social development, and well-being. Am Psychol 2000;55:68-78.

18. Vallerand RJ, Fortier MS, Guay F. Self-determination and persistence in a real-life setting: toward a motivational model of high school dropout. J Pers Soc Psychol 1997;72:1161-76.

19.Williams GC, Saizow RB, Ryan RM. The importance of self-determination theory for medical education. Acad Med 1999;74:992-5. 